\title{
Hepatocarcinoma, porfiria y hemosiderosis. Una asociación no reportada en nuestro país. Caso clínico
}

\author{
CAROLINA WHITTLE ${ }^{1}$, JUAN HEPP ${ }^{2}$, \\ RODOLFO ARMAS ${ }^{3}$, MARCELA SCHULTZ $^{4}$
}

\section{Porphyria cutanea tarda, hemosiderosis and hepatocellular carcinoma. Report of one case}

Porphyria cutanea tarda (PCT) is a hereditary or acquired disease. It can be unleashed by iron overload, alcohol, estrogens and other conditions. In these patients, hepatic involvement can be associated to cirrhosis, iron overload or $C$ and $B$ viral infections, that are predisposing factors for hepatocellular carcinoma. We report a 69-year-old man with PTC, hemosiderosis and hepatocarcinoma. The tumor was diagnosed during a routine ultrasound examination for early detection of malignant lesions. The patient was subjected to a right hepatic excision. The pathological examination of the surgical piece confirmed the diagnosis and disclosed free surgical margins. After 18 months of follow up, the patient had a relapse and a liver transplantation was performed.

(Rev Med Chile 2010; 138: 581-585).

Key words: Carcinoma; Hepatic; Hepatocellular; Hemosidrosia; Porphyrias.

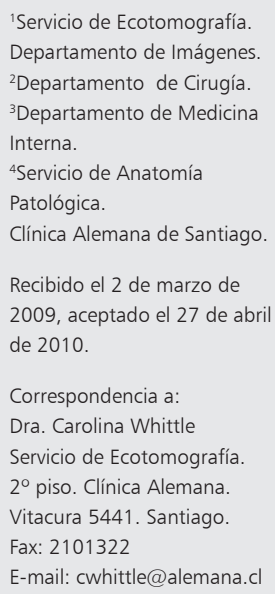

$\mathrm{L}$ as porfirias son enfermedades debidas a desórdenes metabólicos, hereditarios o adquiridos, producidos por un defecto en el control de la biosíntesis del grupo hem. En ellas se acumulan porfirinas o precursores en diversos órganos y aumentan sus niveles en la circulación y en la excreción. En el caso de la variedad cutánea tarda (PCT), éstos se acumulan particularmente en la piel, induciendo efectos citotóxicos y fotosensibilidad con lesiones cutáneas especialmente en áreas de foto exposición.

La PCT puede ser hereditaria o adquirida y ser desencadenada por factores como la acumulación de hierro, el consumo exagerado de alcohol, la administración de estrógenos y las infecciones virales. Frecuentemente la enfermedad es subclínica y se hace evidente cuando se agrega uno o más de los factores desencadenantes. Se ha pensado que la acumulación de Fe en esta afección, pudiera deberse a una asociación genética con la hemocro- matosis, pero esto no se ha demostrado ${ }^{1}$. Setenta por ciento de los pacientes con PCT no tratados tiene niveles elevados de ferritina sérica y 5\% poliglobulia, los que probablemente reflejan el exceso de Fe corporal. Por esto, una de las formas de tratamiento más empleada en ella es el de las sangrías periódicas.

En la PCT ocurren otras asociaciones con patologías que pueden ser causa o efecto de ella. Entre ellas está el compromiso hepático, el que puede estar relacionado con la acumulación de porfirinas en el hígado o con la enfermedad de base que ocasiona o desencadena la PCT como el consumo exagerado de alcohol o la infección con virus hepatotropos. Así, son frecuentes las inclusiones citoplasmáticas aciculares birrefringentes, agregados linfocíticos periductales, depósitos de lipofucsina, infiltración grasa, necrosis focal, inflamación, fibrosis portal, cirrosis y hepatocarcinoma ${ }^{2-4}$. 
Hepatocarcinoma, porfiria y hemosiderosis. Una asociacion no reportada en nuestro país - C. Whittle et al

\section{Objetivos}

Presentar un caso clínico y revisar la literatura sobre la asociación infrecuente de hepatocarcinoma, porfiria cutánea tarda y hemosiderosis.

\section{Caso clínico}

Varón de 69 años, con antecedentes personales y familiares de PCT pesquisados a los 42 años de edad. En ese entonces, presentó hiperpigmentación y erosiones de la piel, principalmente del dorso de las manos, que se producían con los menores traumatismos y que evolucionaban a cicatrices hiperpigmentadas. Como estas alteraciones son típicas de PCT, se evaluó la excreción de porfirinas y sus precursores en el Laboratorio de Gastroenterología del Departamento de Medicina Occidente de la Universidad de Chile, resultando un patrón de excreción característico de PCT (Tabla 1). Se le practicó además biopsia hepática que demostró esteatosis marcada y cirrosis incipiente micro- nodular. No se observó hemosiderosis hepática.

Estudios genéticos moleculares identificaron el genotipo E130D/w en exon 5 del gen uroporfirinógeno decarboxilasa. Por otra parte, estudios de mutaciones del gen HFE mostró que presentaba el genotipo H63D/H63D que está vinculado a la hemocromatosis hereditaria.

El paciente se trató por períodos con flebotomías o con cloroquina, tratamientos de elección para la PCT, desapareciendo rápidamente sus síntomas y alteraciones de laboratorio.

En agosto 2007, en un control médico de rutina estando asintomático, se pesquisó mediante una ecotomografía una imagen compatible con una lesión expansiva hepática de $33 \times 37 \times 39 \mathrm{~mm}$. Una tomografia computada abdominal confirmó la presencia de la masa estimándola de 44 x 40 mm y observó además elementos de cirrosis y cortocircuitos venosos en ligamento falciforme y espleno-renal (Figura 1). La resonancia nuclear magnética mostró reducción de volumen hepático con nódulos de regeneración, aspecto de

Tabla 1. Excreción de porfirinas y sus precursores. Dr. Carlos Wolff (26. 04.1982)

\begin{tabular}{|c|c|c|}
\hline $\begin{array}{l}\text { Porfirinas y sus } \\
\text { precursores }\end{array}$ & Método & Resultados de la evaluación diagnóstica de PCT \\
\hline Excreción urinaria & & Excreción en orina 24 hrs (Valores de referencia) \\
\hline Ácido damino-levulínico & Grabeki (5) & $7,8 \mathrm{mg}(2,13-8,61 \mathrm{mg})$ \\
\hline Porfobilinógeno & Mauzerall \& Granick (6) & $0 ., 62 \mathrm{mg}$ (hasta 1,18 mg) \\
\hline Coproporfirinas & Schwartz \& Zieve (7) & $1285 \mu \mathrm{g}$ (hasta $176 \mu \mathrm{g})$ \\
\hline Uroporfirinas & Schwartz \& Zieve (7) & $4320 \mu \mathrm{g}$ (hasta $15 \mu \mathrm{g}$ ) \\
\hline Excreción fecal & & $\begin{array}{l}\text { Excreción por gr de peso seco de heces (Valores } \\
\text { de referencia) }\end{array}$ \\
\hline Protoporfirinas & Holti \& Rimigton (8) & $120 \mu \mathrm{g}$ (hasta $30 \mu \mathrm{g})$ \\
\hline Coproporfirinas & Holti \& Rimigton (8) & $90 ., 1 \mu \mathrm{g}$ (hasta $20 \mu \mathrm{g}$ ) \\
\hline
\end{tabular}

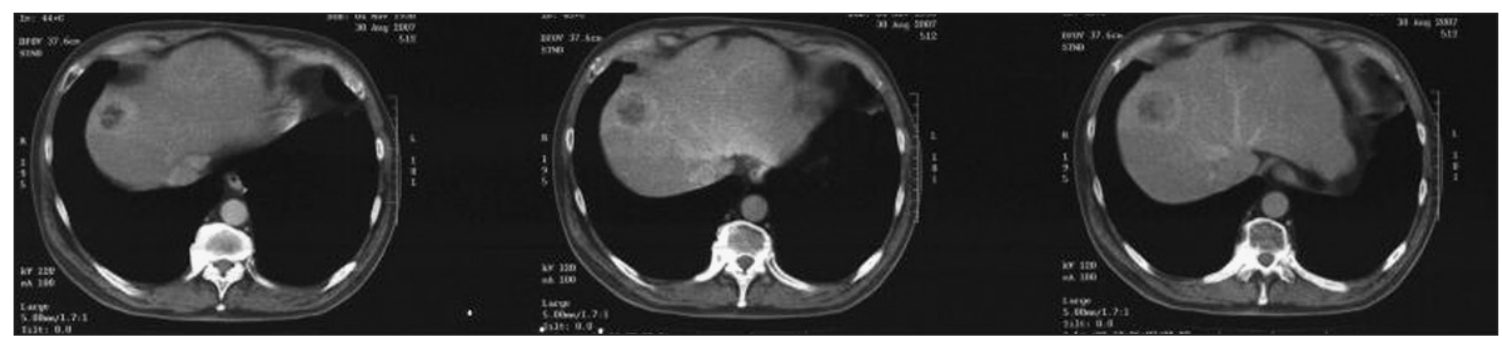

Figura 1. Scanner. Corte con contraste endovenoso muestra lesión tumoral del domo hepático con captación en forma de anillo vascular periférico. 
infiltración por hemosiderina( Figura 2) y tumor del segmento VIII de $40 \mathrm{~mm}$ (Figuras 3). Entre los exámenes practicados, presentaron resultados normales el hemograma, electrocardiograma, bilirrubinemia, protrombinemia y alfa feto proteína; el antígeno de superficie de hepatitis B fue negativo. La transaminasa glutámica pirúvica fue de $109 \mathrm{U} /$ dl y la gamma glutamil transpeptidasa de $144 \mathrm{U} / \mathrm{dl}$.

En base a los antecedentes anteriores, se planteó los diagnósticos de PCT, cirrosis hepática Child Pugh A y de probable hepatocarcinoma derecho de $4 \mathrm{~cm}$. Luego de descartarse mediante estudios de imágenes la presencia de otras localizaciones tumorales, en septiembre de 2007, el paciente fue sometido a una resección hepática derecha (segmentos 4b-5-8). La anatomía patológica

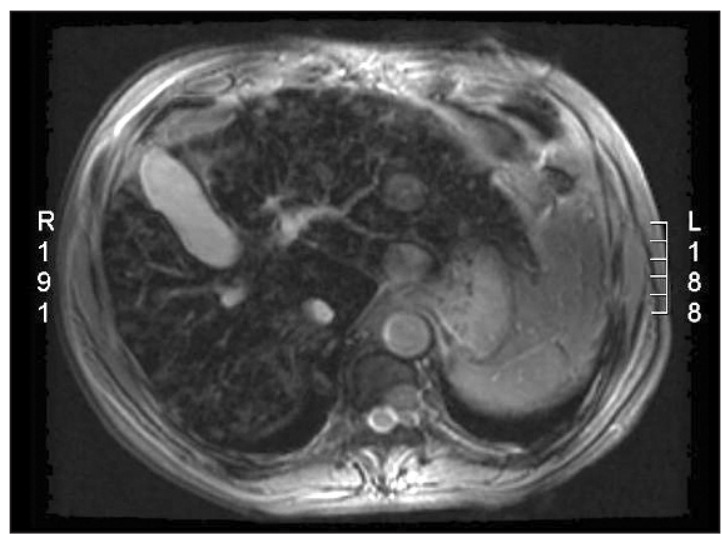

Figura 2. RN secuencia $T 2$ gradiente que demuestra signos de daño por hepatopatía crónica con marcada disminución de la señal del parénquima hepática consistente con sobrecarga de fierro.

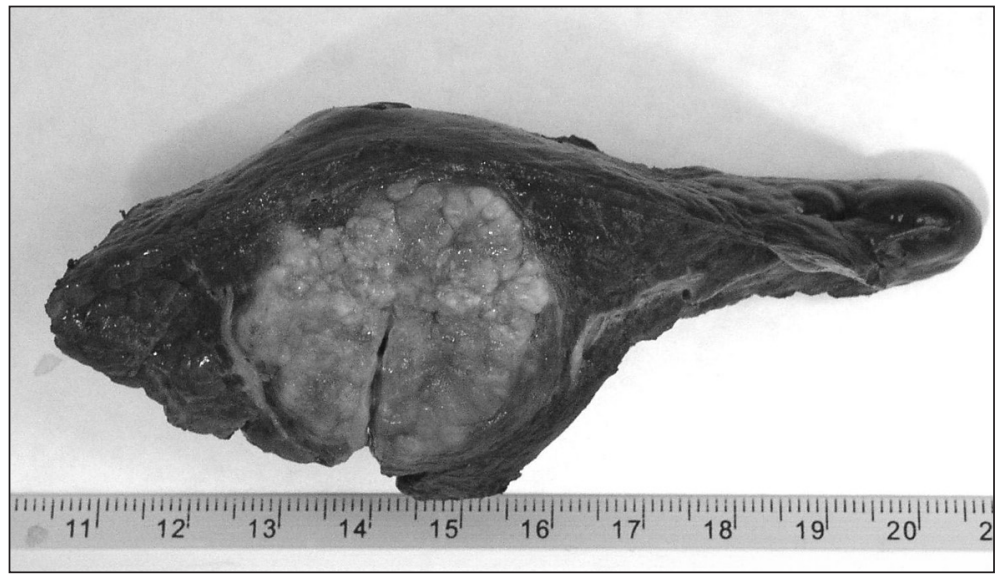

Figura 4. Pieza macroscópica correspondiente a los segmentos IVb , V y VIII con lesión tumoral de $3,7 \mathrm{~cm}$. confirmó un cáncer hepatocelular grado 3 sin evidencias de permeación vascular, cirrosis hepática predominantemente monoacinar con esteatosis macrovacuolar y siderosis grado 3 (Figuras 4, 5, 6). El tumor distaba menos de $1 \mathrm{~mm}$ del borde de la resección quirúrgica en zona profunda y en uno de los bordes laterales de la pieza resecada.

El paciente evolucionó bien y asintomático. En marzo de 2009, en un examen de control, se detectó mediante ecotomografía abdominal presencia de un tumor hepático de $8 \mathrm{~cm}$ y probablemente otro de $4 \mathrm{~cm}$ y, además un trombo portal. Se practicó quimioembolización que produjo una notable reducción del tumor por lo que en julio de 2009 se realizó trasplante hepático que ha evolucionado favorablemente.

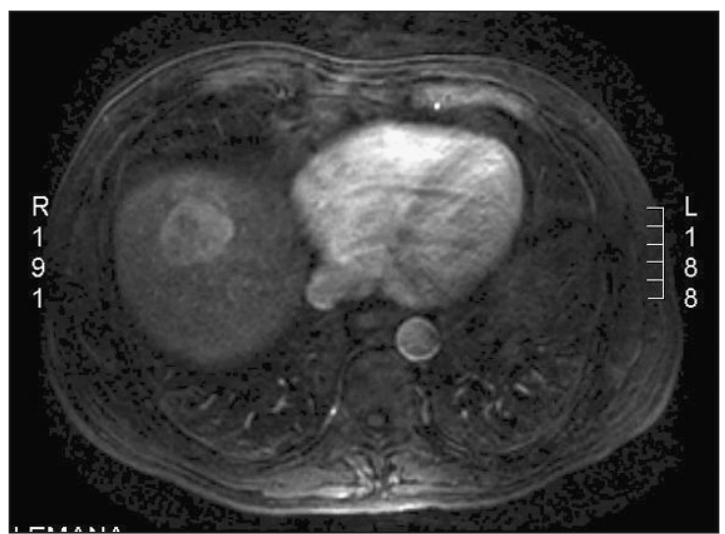

Figura 3. RN secuencia $\mathrm{T} 1$ fat sat con contraste (fase arterial) que demuestra una lesión sólida hipervascular en el domo hepático compatible con hepatoma. 


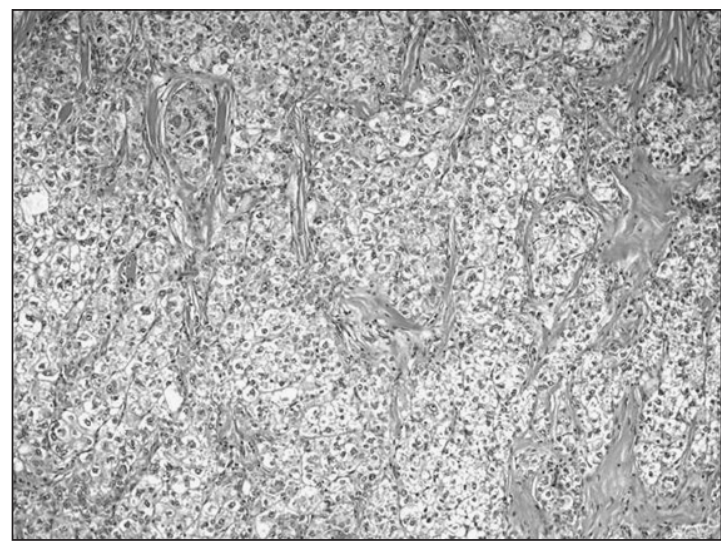

Figura 5. Preparación microscópica teñida con hematoxilina eosina que muestra un hepatocarcinoma con arquitectura trabecular y atipia celular.

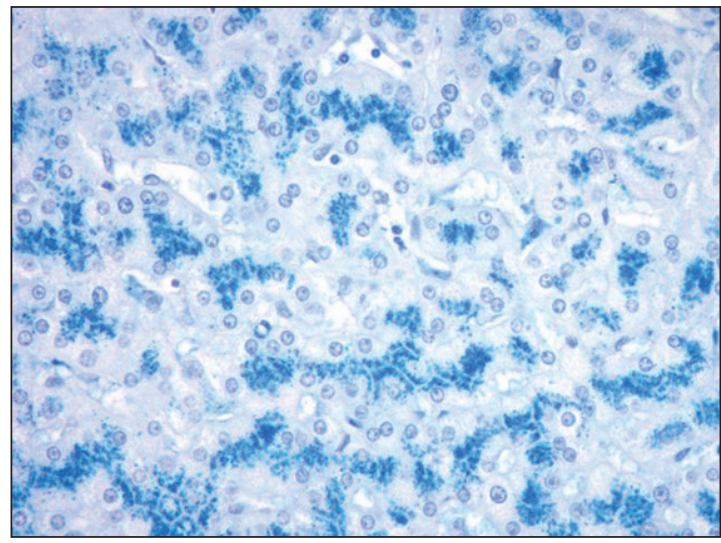

Figura 6. Tinción de azul de Prusia en hígado no tumoral. Se observa depósitos granulares de hemosiderina siguiendo un patrón canalicular.

geográfica, siendo probablemente la PCT la más frecuente en todas las latitudes. Algunas presentan crisis agudas caracterizadas por dolores cólicos abdominales y manifestaciones neuropsíquicas, otras presentan alteraciones cutáneas y otras pueden presentar ambos tipos de manifestaciones. En la PCT sólo hay manifestaciones cutáneas que se presentan predominantemente en el dorso de manos y cara $^{19}$. Esta variedad representaría a $42 \%$ de los casos de porfiria con amplio predominio del sexo masculino ${ }^{19}$. Su forma hereditaria es causada por una mutación del gen uroporfirinogenodecarboxilasa ubicado en la fracción cromosómica 1p34. En forma específica la mutación E130D se ha identificado en otras familias con PCT. La actividad de esa enzima también es bloqueada por el Fe, lo que explica la relación entre PCT y hemosiderosis o hemocromatosis.

La hemocromatosis es una de las enfermedades hereditarias autosómicas recesivas más frecuentes en la población europea caucásica, con una prevalencia de 1:200 a 1:500 personas. Los pacientes presentan una desregulación del metabolismo del Fe con saturación de transferrina elevada y altos niveles de ferritina. La sobrecarga de Fe en los diferentes órganos puede producir manifestaciones clínicas diversas y, entre ellas, precipitar síntomas de PCT en casos en que ésta estaba latente.

Dado la elevada incidencia de hepatocarcinoma en pacientes con cirrosis, es importante contar con métodos de diagnóstico por imagen que permitan detectar precozmente lesiones 
focales y alteraciones del parénquima hepático, especialmente en aquellos casos con PCT. La resonancia magnética puede ser de especial ayuda para diferenciar nódulos displásicos de hepatocarcinoma y además, permite evaluar los depósitos hepáticos de $\mathrm{Fe}^{20}$.

\section{Referencias}

1. Wolff C, Armas Merino R, Frank J, Poblete-Gutiérrez P. Frecuencia de mutaciones del gen de la hemocromatosis en donantes de sangre y en pacientes chilenos con porfiria cutánea tarda. Medicina (Buenos Aires) 2006; 66: 421-6.

2. Armas Merino R, Kause P, Wolff C. Porfiria Cutánea Tarda, Hepatopatía Crónica por virus C y Hepatocarcinoma. Caso clínico. Rev Med Chile 1994; 122: 72-4.

3. Cortés JM, Oliva H, Paradinas FJ, Hernández-Guío C. The Pathology of the liver in Porphyria Cutanea Tarda. Histopathology 1980; 4: 471-85.

4. Salata H, Cortés JM, Enríquez de Salamanaca R, Olivia $\mathrm{H}$, et al. Porphyria Cutanea Tarda and Hepatocellular Carcinoma: Frequency of occurrence and related factors. J Hepatol 1985; 1: 477-87.

5. Grabecki I, Haduch I, Urbanowicz H. Die and enfachen bestmmungsmethoden der D aminolevulinsauer in harm. Int Arch Gewersepath Gewersehyg 1967; 23: 226.

6. Mauzerall D, Granick S. The occurrence and determination of d-aminolevulinic acid and porphobilinogen in urine. J Biol Chem 1956; 219: 435-46.

7. Schwartz S, Zieve L, Watson CJ. An improved method for the determmination of urinary coproporphyrin and an evaluation of factors influencing the analysis. J Lab Clin Med1951; 37: 843-59.

8. Rimington C, Sveinsson SL. The Spectrophotometric determination of uroporphyrin Scand J Clin Lab Invest 1950; 2: 209.

9. Macías Rodríguez MA, Rendó Ucetam P, Tejada Cabrera M, Infante Hernández JM, et al. Factores de riesgo de aparición del carcinoma hepatocelular en pacientes con cirrosis hepática. Rev Esp Enferm Dig (Madrid) 2000; 92: 458-63.

10. Fracazani AL, Taioli E, Sampietro M, Fatta E, Bertelli C,
Fiorelli G, et al. Liver cancer risk is increased in patients with porphyria cutanea tarda in comparison to matched control patients with chronic liver disease. J Hepatol 2001; 35: 498-503.

11. Kordac V. Frequency of occurrence of hepatocellular carcinoma in patients with porphyria cutanea tarda in long term follow-up. Neoplama 1972; 19: 135-9.

12. Siersema FD, Ten Kate FJW, Mulder PG, Wilson JH. Hepatocellular carcinoma in porphyria cutanea tarda: frequency and factors related to its ioccurrence, Liver 1992; 12: 56-61.

13. Mogl, MT, Pascher A. Presser SJ, Schwabe M, Neuhaus P. Neussler NC. An unhappy triad: Hemochromatosis, porphyria cutanea tarda and hepatocellulart carcuinoma. A case report. World J Gastroenterol 2007; 13: 1998-2001.

14. Cauza E, Peck-Radosavijevic M, Ulrich-Pur H, Datz $\mathrm{C}$, et al Mutations of the HFE gene in patients with hepatocellular carcinoma. Am J Gastroenterol 2003; 98: 442-7.

15. Boige V, Castera L, de Roux N, Ganne-Carrie N, Ducot B, Pelletier G, et al. Lack of association between HFE gene mutations and hepatocellular carcinoma in patients with cirrhosis. Gut 2003; 52; 1178-81.

16. Bjersing L, Andersson C, Lithner F. Hepatocellular carcinoma in patients from northern Sweden with acute intermittent porphyria: morphology and mutations. Cancer epidemiology, biomarkers \& prevention 1996; 5; 393-7.

17. Andersson C, Bjersing L, Lithner F. The epidemiology of hepatocellular carcinoma in patients with acute intermitent porphyria. A population-based study. J Intern Med 1996; 240: 195-201.

18. Andant C, Puy H, Faivre J, Deybach JC. Acute hepatic porphyrias and primary liver cancer (Letter). N Engl J Med 1998; 338: 1853-4.

19. Armas Merino R, Wolff C, Krause P, Chana P, Parraguez A, Soto JR. Las porfirias hepáticas: experiencia con 105 casos. Rev Med Chile 1992; 120: 259-68.

20. Katsuyoshi I, Mitchell DG, Gabata T, Hann HL, et al. Hepatocellular Carcinoma: association with increased iron deposition in the cirrhotic liver at MR imaging. Radiology 1999; $212: 235-40$. 